\title{
THE DISPERSIONS OF NANOPARTICLES IN WATER-ORGANIC SOLVENTS AS THE BASIS FOR THE SILVER NANO-INK FOR INK- JET PRINTING
}

\author{
Sergey V. Tkachev, Sergey P. Gubin \\ "AkKo Lab" LLC, http:/ / akkolab.ru.en \\ 129110 Moscow, Russian Federation \\ Kurnakov Institute of General and Inorganic Chemistry, Russian Academy of Sciences, http:/ /www.igic.ras.ru \\ 117991 Moscow, Russian Federation \\ tkachev_svmsu@mail.ru,gubin@igic.ras.ru
}

Vitalii P. Kim, Alexey E. Kushnir, Denis Yu. Kornilov

"AkKo Lab" LLC, http://akkolab.ru.en

vp.kim@physics.msu.ru, kushnir.a.e@gmail.com, kornilovdenis@rambler.ru

Abstract. The work is aimed to solve the problem of developing functional silver nano-ink applied in ink-jet technology in printed flexible electronics, as well as to demonstrate printing results and their discussion. The development of functional compounds for printing is a fundamentally new approach to the production of flexible electronic devices for the military and civil industry. As a part of the work special attention is paid to the preparation of aqueous and organic dispersions of silver nanoparticles, the study of the various factors which affect the size and characteristics of the nanoparticles, methods of concentrating the standard dispersions to obtain nano-ink, printing the conductive structures by synthesized nano-ink and research of the printed elements' properties. These results give an opportunity to develop silver nano-ink and adapt it for printing on various substrates (including flexible polymeric ones) by specialized equipment.

Keywords: silver nano-ink, aqueous dispersions, ink-jet printing, conductive structures, polymeric substrate, flexible printed electronics

UDC 546.57, 621.3.049.7

Bibliography -8 references

Received 5.12.2016

RENSIT, 2016, 8(2):171-184

DOI: $10.17725 /$ rensit.2016.08.171

CONTENTS

1. INTRODUCTION (171)

2. THE EXPERIMENTAL SECTION (174)

2.1. Preparation of dilute aqueous DISPERSIONS OF SILVER NANOPARTICLES (174)

2.2. Preparation of Silver Nano-INK (174)

3. Results and Discussion (174)

3.1. INK-JET PRINTING WITH USE OF SILVER NANO-INK (176)

3.2. Treatment of substrates IN PLASMA (177)

3.3. Thermal treatment (178)

3.4. Printing of the conductive lines ON THE ITO SURFACE WITH THE USE OF SILVER NANO-INK (178)
3.5. Printing of the Conductive Lines ON THE SILICON SURFACE WITH THE USE OF SILVER NANO-INK (180)

3.6. Printing of the heating elements ON THE POLYMER FILM (181)

3.7. Printing of the complexGEOMETRY CONDUCTIVE BOARDS ON DIFFERENT SUBSTRATES (181)

3.8. Printing on textile goods With THE USE OF SILVER NANO-INK (182)

4. Conclusion (182)

ReferenCEs (183)

\section{INTRODUCTION}

Printed flexible electronics is a new perspective direction on development of microelectronic flexible devices by the printing additive 
technologies of the next generation including ink-jet printing [1]. Nowadays major foreign corporations, interested commercial organizations and separate research groups are working at creation and development of flexible electronic devices by the method of precision additive printing with the use of specialized printers.

Intensive development of the mentioned direction in microelectronics is caused by sharp monthly increase in the number of scientific publications devoted to creation of printing microelectronic devices.

Use of the additive printing is urgent due to the following advantages: increasing technological capabilities to use the flexible electronic devices; simplification of technological cycle of design and manufacture of electronic circuit boards; reduction in the number of technological operations; reduce in energy costs; significant improvement of the environmental safety of the technological process.

Nowadays considerable work of scientists and designers in the sphere of printed and flexible electronics is aimed at development of new functional materials - nano-ink which would give an opportunity to excel the corresponding solid state electronic analogs produced with the use of the traditional methods in some crucial parameters. Thus, depending on the aim of use, nano-ink for special purposes is being developed. They are the following: the conductive on the basis of metal nanoparticles (mainly silver nanoparticles), semiconductive, magnetic and carbon ones on the basis of graphene and related nanoobjects and nanotubes [2-3]. It should be noted that the majority of printed electronic tasks require silver nano-ink.

Thus, this article presents the results of developments of the "AkKo Lab" LLC company and their discussion. They include creation of silver nano-ink adapted for printing on the available high-technology equipment Dimatix Materials Printer 2831 and also the results of various structures' printing on the different-nature substrates.

In terms of chemistry, silver nano-ink is highly concentrated dispersions of silver nanoparticles. As a rule, mass content of silver nanoparticles in such systems accounts for more than $5-10 \mathrm{wt}^{\mathrm{O}} \%$. It is obvious that synthesis of dilute dispersions of silver nanoparticles is quite well-developed nowadays, and there are a great number of various methods and options to obtain them. However, there are some problems related to production of highly concentrated dispersions of silver nano-ink applied particularly to print the conductive lines by ink-jet printing method. Besides that, to use nano-ink in cartridges of the ink-jet printers Dimatrix, the following requirements should be fulfilled: the used nano-ink should have viscosity 10-20 cP, surface tension 2833 dynes/cm and $\mathrm{pH}$ 4-9, particle size (preferably less than $50 \mathrm{~nm}$ ) ensuring successful ink's passage through the nozzles of the size about $17 \mu \mathrm{m}$ and also absence of harmful and poisonous substances in nanoink composition.

It is also necessary to notice that the nanoink use in printing on the basis of various nanoobjects is justified by the precision microprint and the unique properties of nanomaterials in comparison with similar compact substances. It is worth noticing that nowadays on the world market there is only some simpler nano-ink (according to its composition), mainly silver one [4]. As a rule, the main difficulties at synthesis of nano-ink adapted for ink-jet printing are the following: stabilization of individual nanoparticles 
at increase in their concentration in dispersion (up to several tens of mass percentage of the substance), achievement of the required surface tension, nano-ink viscosity. These difficulties are being solved by not only selection of the conditions for synthesis realization (duration, temperature, components concentration), but also by choice of various kinds of surfactants, solvents. In general, the great number of methods is original because nano-ink is a complex multi-component solution.

The work innovation is in the development of new, original according to their composition, highly concentrated dispersions of functional silver nanoparticles which allow producing elements of flexible microelectronic devices by the method of ink-jet printing.

Nowadays such large companies as "Fujifilm", "Novacentrix", "Ceradrop" and the others produce and supply the printers capable to print pictures of any geometry using nano-ink. Moreover, refill of the corresponding cartridge is possible using any nano-ink meeting the printing requirements; the substrates choice is not limited. Now printing accuracy of such printers does not exceed several microns, and minimum size of an individual drop is more than $15 \mu \mathrm{m}$. This is due to the sizes of the cartridge nozzles, surface tension and nano-ink viscosity, the adhesive properties of the substrate and precision of the stepper motors of the printer. Nowadays, using such printed installations and nano-ink, specializing companies and individual research teams print the majority of microelectronics' element base: capacitors, accumulators, conductive lines, LEDs and the others [5-7]. Besides, some experiments on printing LEDs, transistors on the flexible transparent polymer substrates are described in literature. Large-scale production with the application of printing additive technologies is possible using roll-to-roll technology where the roll of the required material is applied as a substrate. This technology is suitable to print and carry out the following temperature (thermal, light, etc.) treatment of electronic devices with the feed rate of the substrate as several meters per a minute.

Combination of functional nanoink (with characteristics of conductors, semiconductors and dielectrics) with the modern printed installations may reduce the cost of the electronic products significantly, increase efficiency of their production, give an opportunity to produce flexible electronic devices.

Ink-jet printing technology differs from the traditional technologies of electronic industry (photolithography, etc.) in the low production cost, possibility to work with any substrate type, and substrates in this technology do not experience significant impacts. Thus, the technology gives an opportunity to produce flexible transparent electronic devices with relatively low cost. The final product in the ink-jet printing is batteries, accumulators, capacitors, thin-film transistors, displays, sensors, antennas, solar batteries, the connecting elements in chips, etc. [8]

The problem statement in this work is the need to arrange mass production of silver nano-ink which is stable during storage and transportation and suitable for ink-jet printing of the conductive structures on the various substrates (including flexible ones) using modern $2 \mathrm{D}$ printers.

The article shows how to solve the assigned task by the approaches and methods of colloidal chemistry, gives the results 
of some elements' printing, presents their measured characteristics.

\section{EXPERIMENTAL SECTION}

The initial reagents for the silver nano-ink synthesis were silver nitrate (chemically pure, «Bertuz» JSC), silver acetate ( $\geq 99.0 \%$, CASNo.: 563-63-3, Sigma Aldrich), tri-sodium citrate 2-hydrate $(99.0 \%$, CAS-No.: 6132 04-3, Panreac), sodium borohydride (96\%, CAS-No.: 16940-66-2, Panreac), ethylene glycol (analytically pure, CAS-No.: 107-21-1, «Ekos-1» JSC) and deionized water.

\subsection{Preparation of dilute aqueous DISPERSIONS OF SILVER NANOPARTICLES}

In a typical synthesis, $4 \mathrm{~g}$ of sodium citrate was dissolved in deionized water in a flatbottomed flask at continuous stirring. Next, a solution containing $0.5 \mathrm{~g}$ of silver nitrate and silver acetate in deionized water was added to sodium citrate solution in small portions at constant stirring. Additionally $0.2 \mathrm{M}$ aqueous sodium borohydride solution was prepared. Further, freshly prepared sodium borohydride solution was added dropwise to a solution containing sodium citrate, nitrate and silver acetate. The obtaining dispersion of silver nanoparticles acquired an intense dark brown color. As a result, the standard aqueous dispersions of silver nanoparticles containing $0.03 \mathrm{wt} \%$ silver nanoparticles were obtained.

\subsection{Preparation of Silver nano-InK}

To synthesize silver nano-ink, the standard dispersion of silver nanoparticles was centrifuged at $15000 \mathrm{rpm}$ for $20 \mathrm{~min}$. Further the supernatant was decanted and the obtaining highly concentrated liquid of silver nanoparticles was collected. Then centrifugation step was repeated again. Further surfactants were added to the resulting silver nanoparticles concentrate. They included ethylene glycol which is needed to stabilize the obtained silver nanoparticles. Then, the obtained highly concentrated dispersion of the nanoparticles in ethylene glycol was passed through the filter with a pore size of $0.22 \mu \mathrm{m}$. As a result, the targeted silver nano-ink was obtained.

The morphology, composition and structure of the obtaining nano-objects in the work were examined by scanning electron microscopy SEM (the scanning probe microscope Carl Zeiss Supra 40- 30- 87, Germany), atomic force microscopy AFM (the atomic force microscope Solver P47, «NT-MDT» Co, Russia), transmission electron microscopy TEM (the transmission electron microscope JEOL 2000FX, Germany), the powder X-ray diffraction XRD (the diffractometer Bruker Advanced 8 at $\mathrm{CuK \lambda}$ radiation and $\lambda=1,5418 \AA$ ), spectrophotometry (the spectrophotometer Leki SS2107UV, «LOIP» JSC, Russia)

\section{RESULTS AND DISCUSSION}

It should be noted that the structure and the spectral properties of nanoparticles depend significantly not only on composition and the characteristics of the dispersion where they are dispersed but also on the synthesis conditions, use of one or another stabilizer, solvent, concentration of initial reagents, etc. Due to the fact that nanoparticles are generated using standard methodologies, it is still being discussed if it is possible to keep the composition, structure and specific optical physical characteristics of initial nanoparticles when they become final product - highly concentrated dispersions of these nanoparticles - nano-ink. The authors would like to confirm the possibility to prepare silver nano-ink from standard silver dispersions 
in aqueous dispersion keeping their main characteristics and also to show and discuss the results of conductive structures' printing with the use of synthesized nano-ink on the printer Dimatix Materials Printer 2831.

Figure 1 presents typical absorption spectra depending on wavelength for diluted samples of standard dispersion of silver nanoparticles and nano-ink produced from the given dispersion. Absorption extremum takes place at wavelength of $388 \mathrm{~nm}$ which corresponds to the average nanoparticles size of $10 \mathrm{~nm}$, according to the literature data.

Spectral analysis shows that operations of centrifugation and concentration of standard dispersion of silver nanoparticles lead to not only increase in concentration of the targeted nanoparticles in nano-ink but more noticeable monodispersion of the end product in comparison with initial dispersion.

According to the data of XRD analysis, reflections of crystal structure of silver nanoparticles isolated both from standard dispersion of silver nanoparticles and from silver nano-ink and after that prepared at $100^{\circ} \mathrm{C}$ are equal to the phase of massive silver (the card PDF\#030921, database of the International Centre for Diffraction Data).

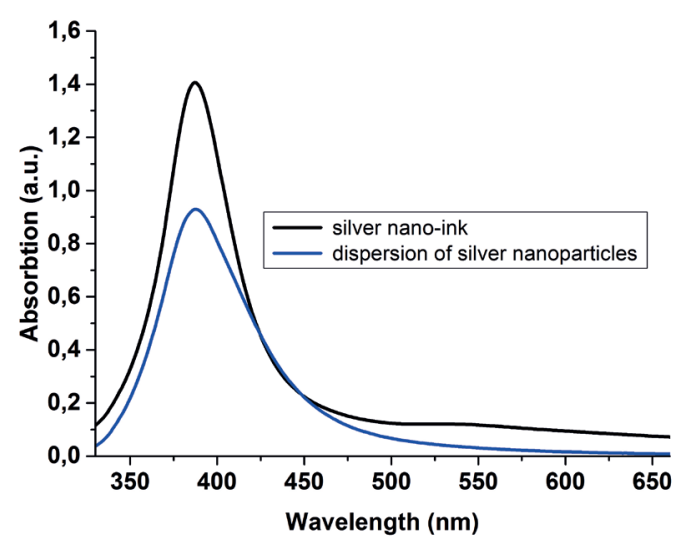

Fig. 1. Typical absorption spectra of diluted dispersions of silver nanoparticles and nano-ink.

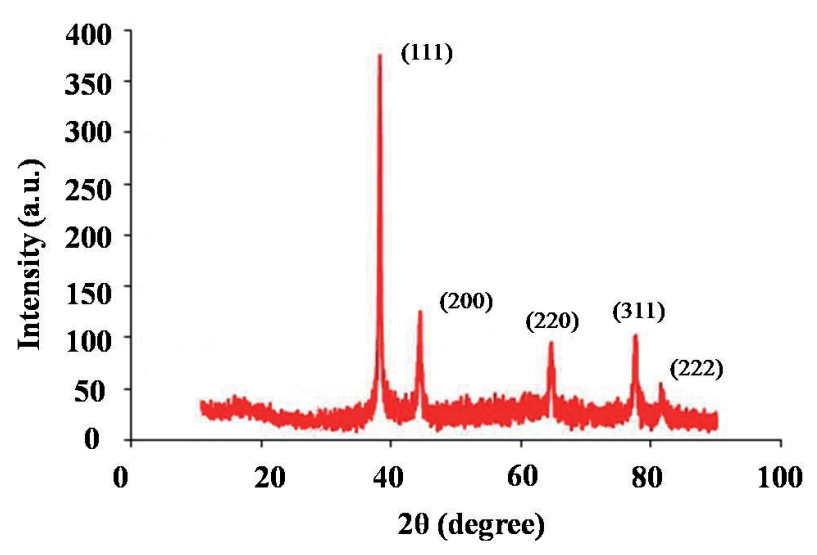

Fig. 2. XRD pattern of silver nano-ink produced by thermal treatment of silver inks' aliquot at temperature $100^{\circ} \mathrm{C}$ for $30 \mathrm{~min}$.

Figure 2 gives diffraction pattern of silver nano-ink produced by thermal treatment of silver inks' aliquot at temperature $100^{\circ} \mathrm{C}$.

Analysis of silver nano-ink samples by the transmission electron microscopy method shows that dispersions of silver nanoparticles and nano-ink consist of isotropic nanoparticles (Figure 3). It should be noticed that the particles' shape is quite uniform and close to the spherical one. According to the results of the electrondiffraction pattern obtained by TEM method, silver nano-ink has crystal nature. Thus, ringshaped reflections on the electron-diffraction pattern identify the phase of compact silver and the sample's single-phase state.

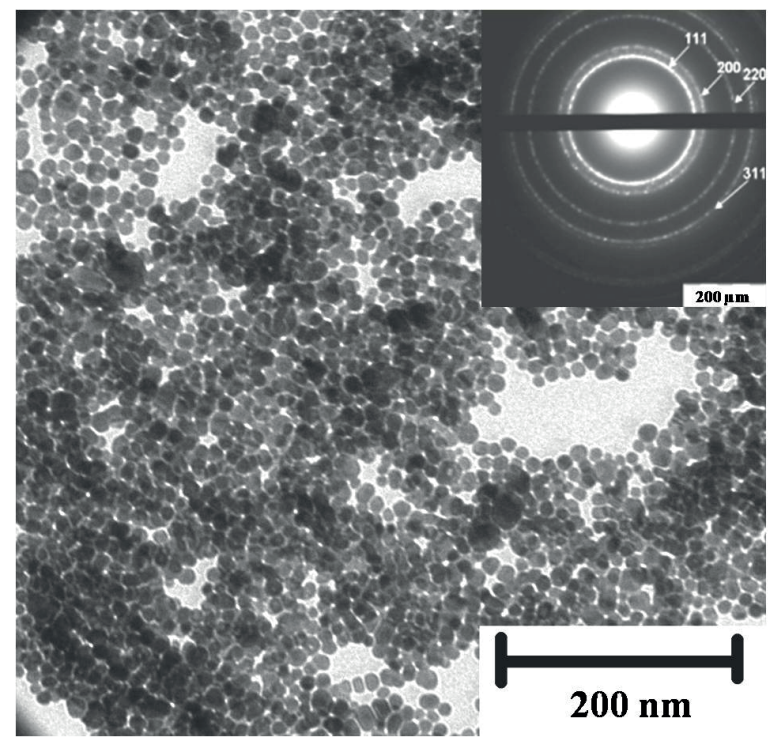

Fig. 3. TEM-image and electron diffraction of silver nanoparticles from silver nano-ink. 


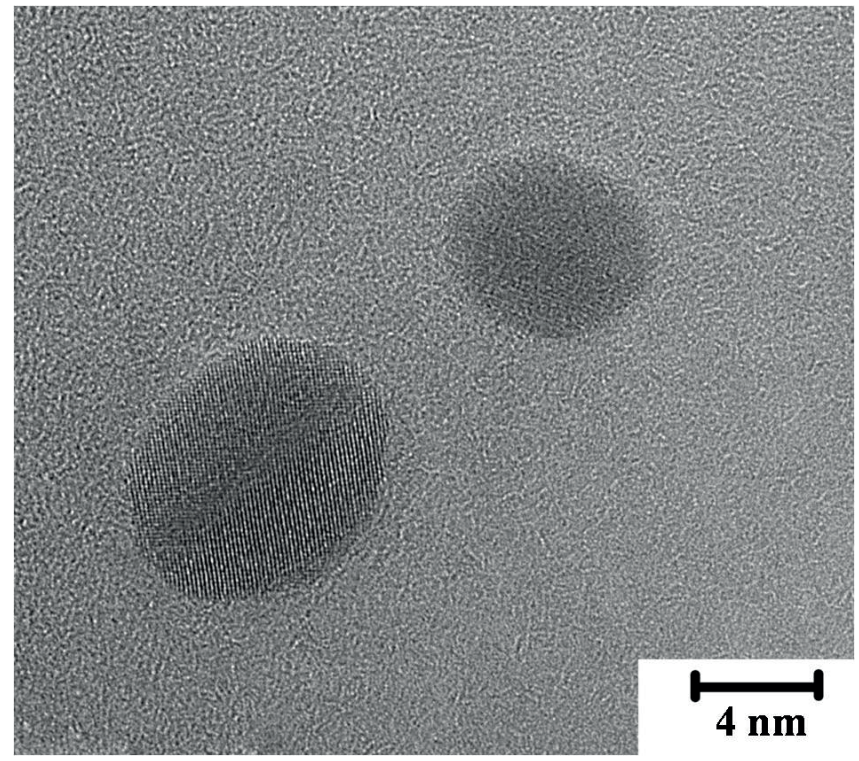

Fig. 4. HRTEM - high resolution image of silver nanoparticles from silver nanoparticles' dispersion.

It is shown that the results obtained using TEM technique correspond with the spectrophotometry results.

Figure 4 presents the high resolution transmission electron microscopy (HRTEM) for two silver nanoparticles. The structure and the shape of nanoparticles are distinct in the picture.

According to the histogram (Figure 5) of silver nanoparticles' distribution at sizes, it was stated that the average silver nanoparticles size accounts for $10 \pm 2 \mathrm{~nm}$ and the range of

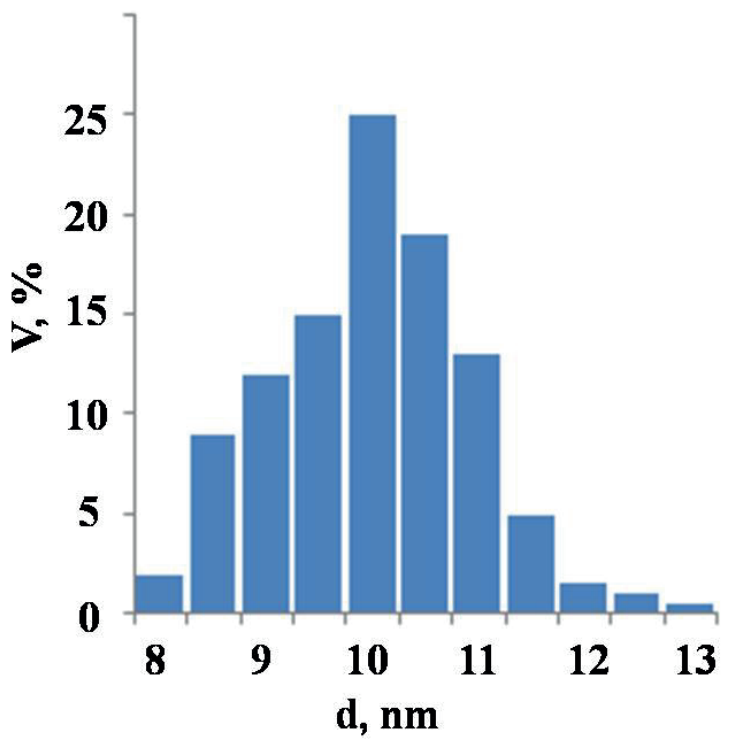

Fig. 5. The histogram of silver nanoparticles' distribution at sizes. nanoparticles distribution at sizes is quite narrow.

Some physical properties of the produced nano-ink were investigated. Thus, viscosity of synthesized silver nano-ink accounted for $17-20 \mathrm{cP}$, surface tension $-28-30 \mathrm{mN} / \mathrm{m}$.

Thereby, standard dispersions of silver nanoparticles and silver nano-ink were studied and characterized. It was ascertained that the developed silver nano-ink met the requirements for $2 \mathrm{D}$ ink-jet printing completely.

\subsection{INK-JET PRINTING WITH USE OF SILVER NANO-INK}

Ink-jet printing by synthesized nano-ink was implemented using the printer (Fujifilm Dimatix Inc) Dimatix DMP-2831 equipped with the 16-nozzles cartridge and the silicon MEMS printer head; a nominal drop volume of every nozzle is up to $10 \mathrm{pl}$ (Figure 6).

The main printing parameters are the volume of the ink drop formed in the printer's cartridge nozzle and the distance between two next drops on the substrate. The drop's volume - up to $10 \mathrm{pl}$ - is optimal due to the use of silver nano-ink with the size up to $12 \mathrm{~nm}$ mainly. At this drop volume, the lateral drop size on the substrate's surface does not exceed $20 \mu \mathrm{m}$ which characterizes print resolution at this method.

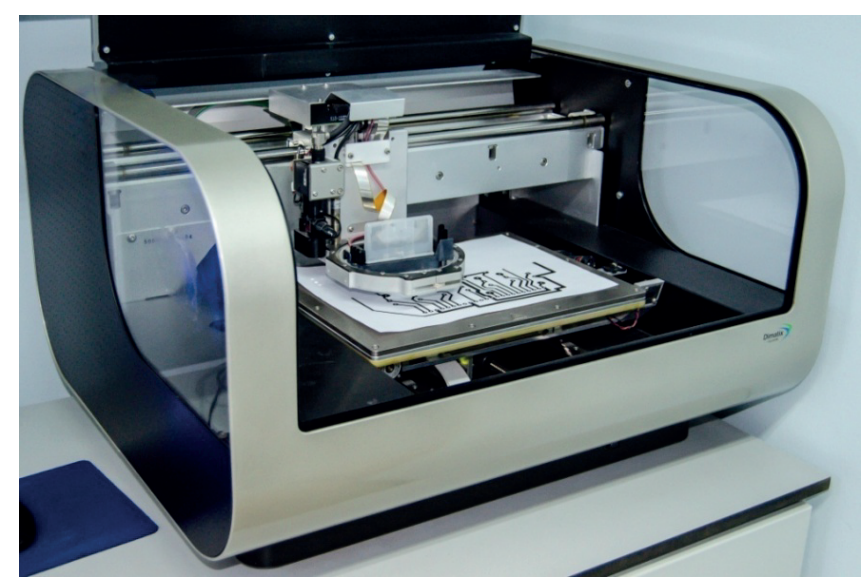

Fig. 6. Ink-jet printing equipment - Fujifilm Dimatix-2831. 


\section{NANOSYSTEMS}

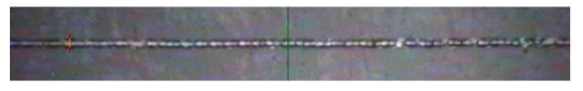

3 layers

$(25 \mu \mathrm{m})$

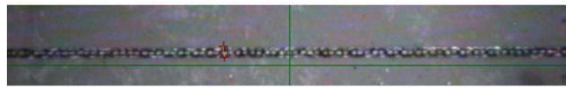

5 layers

$(31 \mu \mathrm{m})$

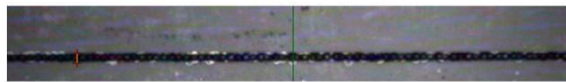

10 layers

$(25 \mu \mathrm{m})$

20 layers

$(31 \mu \mathrm{m})$

Fig. 7. Image of the lines with different number of layers printed by silver nano-ink on the polymer film; the image was obtained using the optical microscope (the line width is given in brackets).

In order to obtain continuous lines, printing may be done in several passes. Figure 7 shows the lines printed using silver nano-ink with different number of layers, printing was done 3; 5; 10 and 20 times. However, the printed line widened insignificantly: from $25 \mu \mathrm{m}$ to $31 \mu \mathrm{m}$.

Figure 8 shows a photograph of the printed silver lines on flexible polymer films. The width of the narrowest line is $30 \mu \mathrm{m}$.

\subsection{Treatment of Substrates IN Plasma}

As the applied printed technology is based on ink-jet principle, one of conditions for printing is suitable surface characteristics of the substrates, particularly the contact wetting angle (or limiting wetting angle) of the substrate surface. If the substrate surface is hydrophobic, shrinking of the printed structure into individual drops may take place which is the reason for crippling of the printed structures. To solve the task of the substrates hydrophilization,

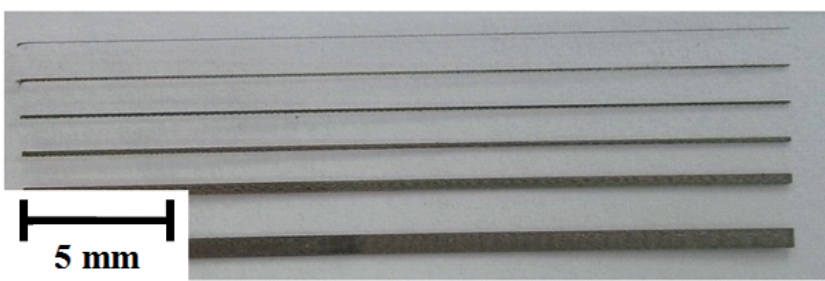

Fig. 8. The photograph of the printed silver lines on flexible polymer films. The width of the narrowest line is $30 \mu \mathrm{m}$.
THE DISPERSIONS OF NANOPARTICLES IN WATER-ORGANIC 177 SOLVENTS AS THE BASIS OF THE SILVER NANO-INKS...

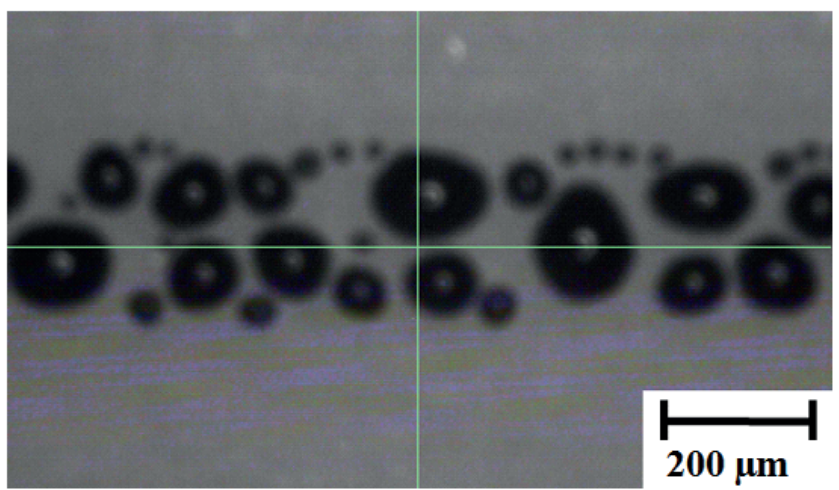

Fig. 9. Image of the $200 \mu m$-width line printed by silver nano-ink on the polyimide film; image is obtained by optical microscopy method.

treatment of the substrate in plasma is applied. Thus, experiments on plasma influence of surface characteristics of ceramic and polyimide substrates were carried out using the equipment "Tantec VacuLAB". At printing, nano-ink formed isolated drops on the substrates which had not been treated in plasma (Figure 9).

If plasmainfluencesceramicand polyimide substrates, contact wetting angle of the latter one increases, surface characteristics become hydrophilic. Figure 10 shows the printed lines on the plasma-treated surfaces.

Thus, the presented technology of the surfaces hydrophilization widens the range of the substrates' types applied in the technology of ink-jet printing of the electronic components.

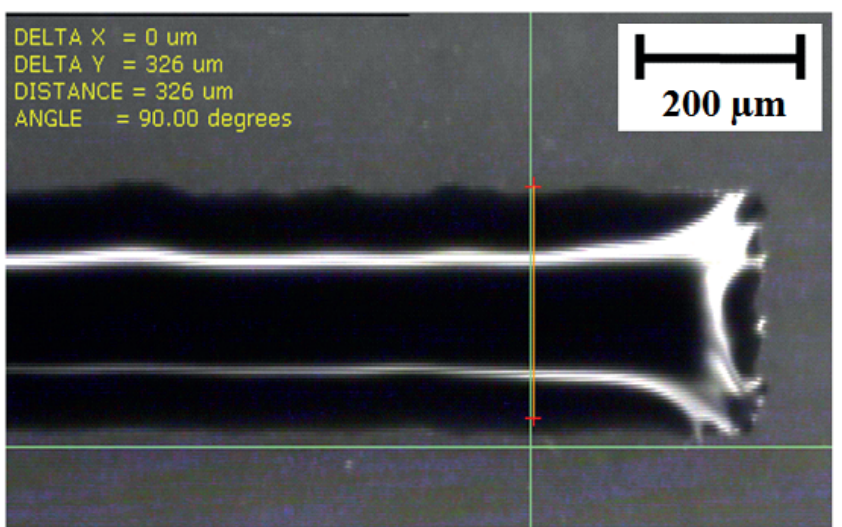

Fig. 10. Image of the $200 \mu m$-width line printed by silver nano-ink on the polyimide film; the image is obtained by optical microscopy method. 


\subsection{Thermal treatment}

To bond the individual silver nanoparticles and then to form the single metal structure, it is necessary to heat particles by means of thermal treatment or high-energy optical pulses.

Figure 11 presents the images of scanning electron microscopy of the lines printed by silver nano-ink at different annealing temperature.
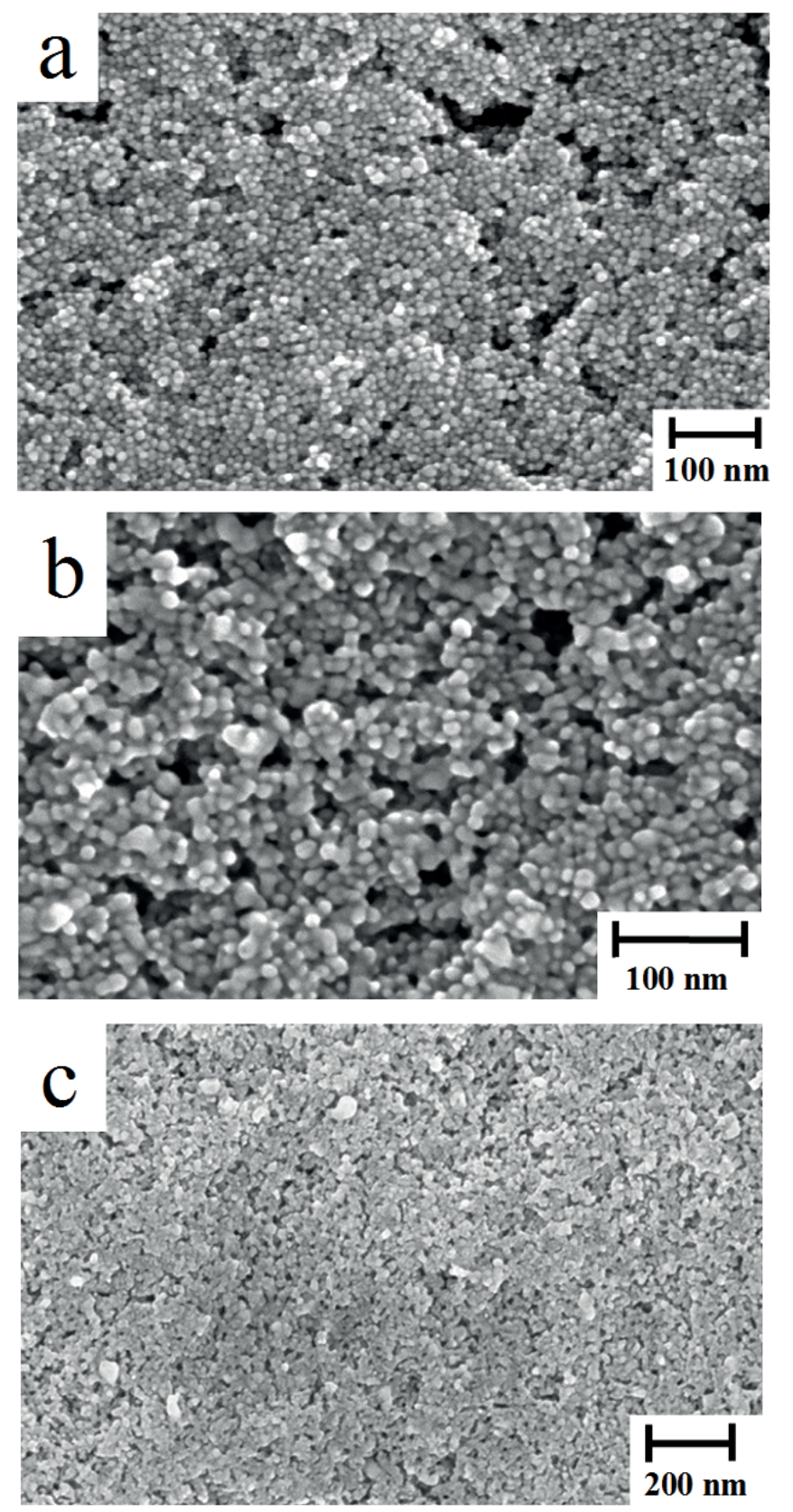

Fig. 11. SEM-images of silver nano-ink: a - without thermal treatment; $b$ - thermal treatment at $90^{\circ} \mathrm{C} ; c$ - thermal treatment at $150^{\circ} \mathrm{C}$.
It should be noticed that melting point of massive silver is $962^{\circ} \mathrm{C}$. The given pictures show that silver nanoparticles start sintering between each other at $90^{\circ} \mathrm{C}$. The reason for this is the heightened surface energy of nanoparticles. Taking into consideration that such heating (about $90^{\circ} \mathrm{C}$ ) does not destroy the majority of polymeric films, this technology may be successfully applied to create the metal conductive structures on the flexible polymer films.

Measurements of resistivity of the structures printed by silver nano-ink were carried out at different annealing temperatures (Figure 12).

The result is that the value of silver nano-ink resistivity at annealing temperature $400^{\circ} \mathrm{C}$ accounts for $0.025 \mathrm{Om} \cdot \mathrm{mm}^{2} / \mathrm{m}$. Meanwhile, the value of massive silver is $0.015 \mathrm{Om} \cdot \mathrm{mm}^{2} / \mathrm{m}$. This confirms almost complete sintering of nanoparticles massive into the single structure.

\subsection{Printing of the conductive lines on} THE ITO SURFACE WITH THE USE OF SILVER NANO-INK

Printing by silver nano-ink was implemented on the silicon substrate covered by ITO layer with the use of the Fujifilm Dimatix 2831 printer. The ITO - Indium Tin Oxide - layer

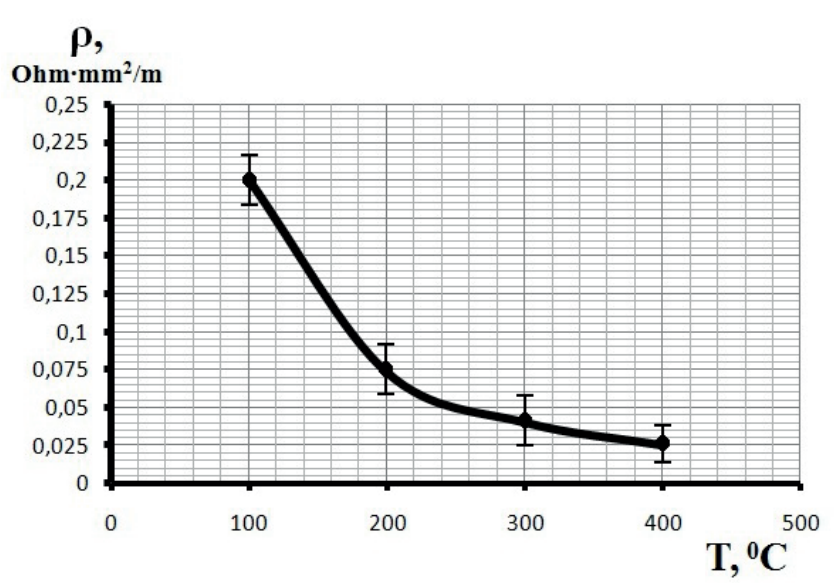

Fig. 12. The plot of resistivity of the structures printed by silver nano-ink at annealing temperature from $100^{\circ} \mathrm{C}$ to $400^{\circ} \mathrm{C}$. 


\section{NANOSYSTEMS}

a
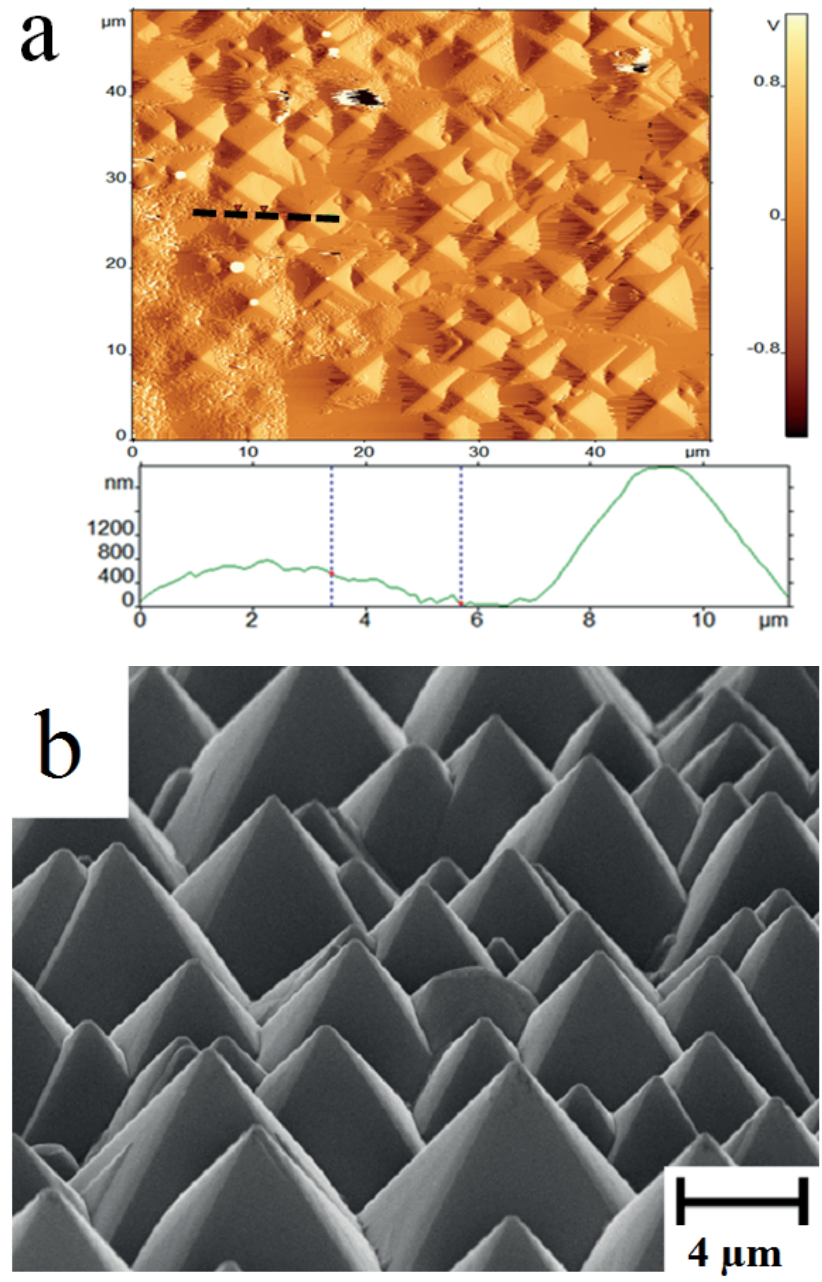

Fig. 13. Images of ITO surface: $a-a$ top view (obtained by the AFM method), $b-a$ side view (obtained by the SEM method).

consists of $90 \mathrm{wt}^{0} \% \mathrm{In}_{2} \mathrm{O}_{3}$ and $10 \mathrm{wt}^{0} \% \mathrm{SnO}_{2}$. The ITO layer is optically transparent and electroconductive.

Surface of the given substrates and the structure of the printed lines were investigated by the scanning electron microscopy and atomic force microscopy (Figures 13-16).

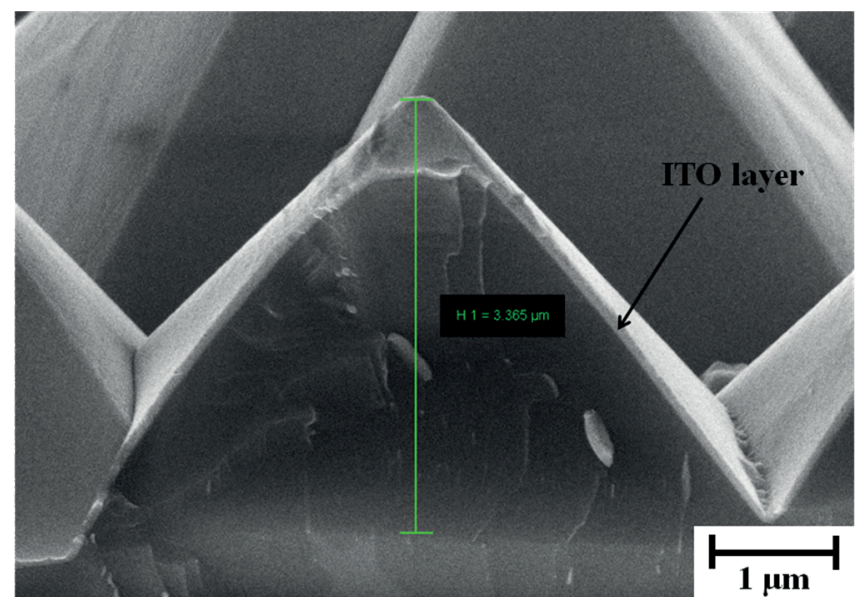

Fig. 14. SEM - image of the surface cleavage.
THE DISPERSIONS OF NANOPARTICLES IN WATER-ORGANIC $\mathbf{1 7 9}$ SOLVENTS AS THE BASIS OF THE SILVER NANO-INKS...
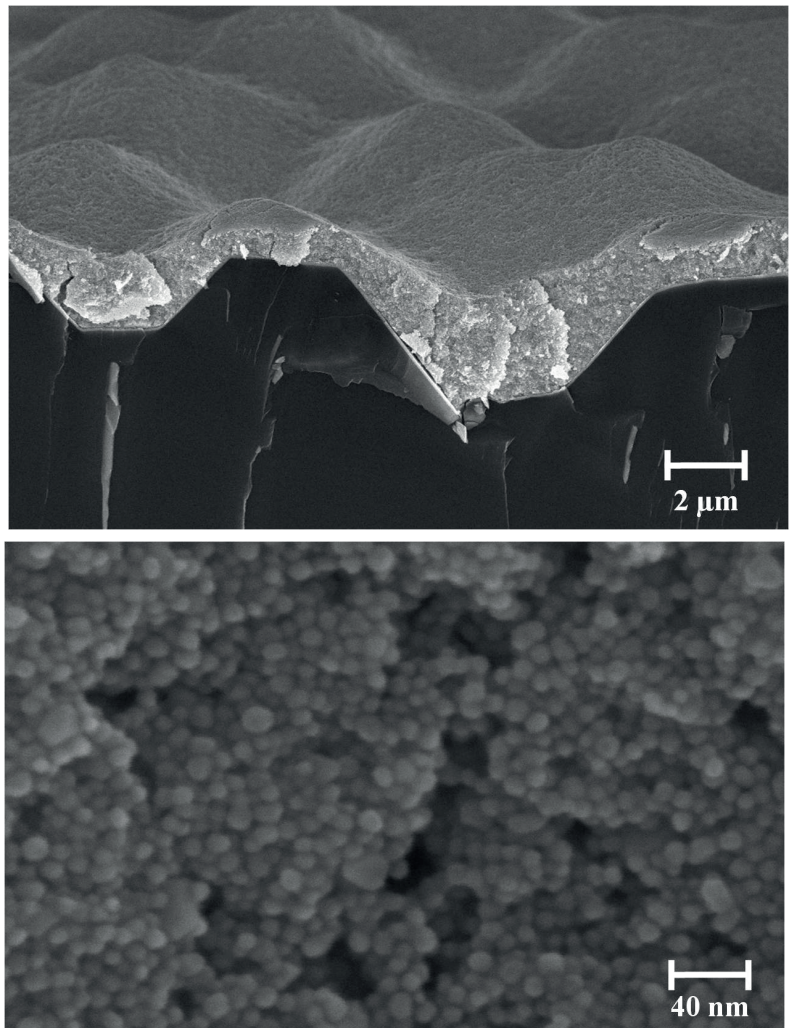

Fig. 15. SEM-images of the printed annealed conductive elements on the ITO surface: at the top - a side view, on the down - a top view.

The width of the printed silver conductive lines is from $100 \mu \mathrm{m}$ up to $2 \mathrm{~mm}$, and the height of the obtained structures does not
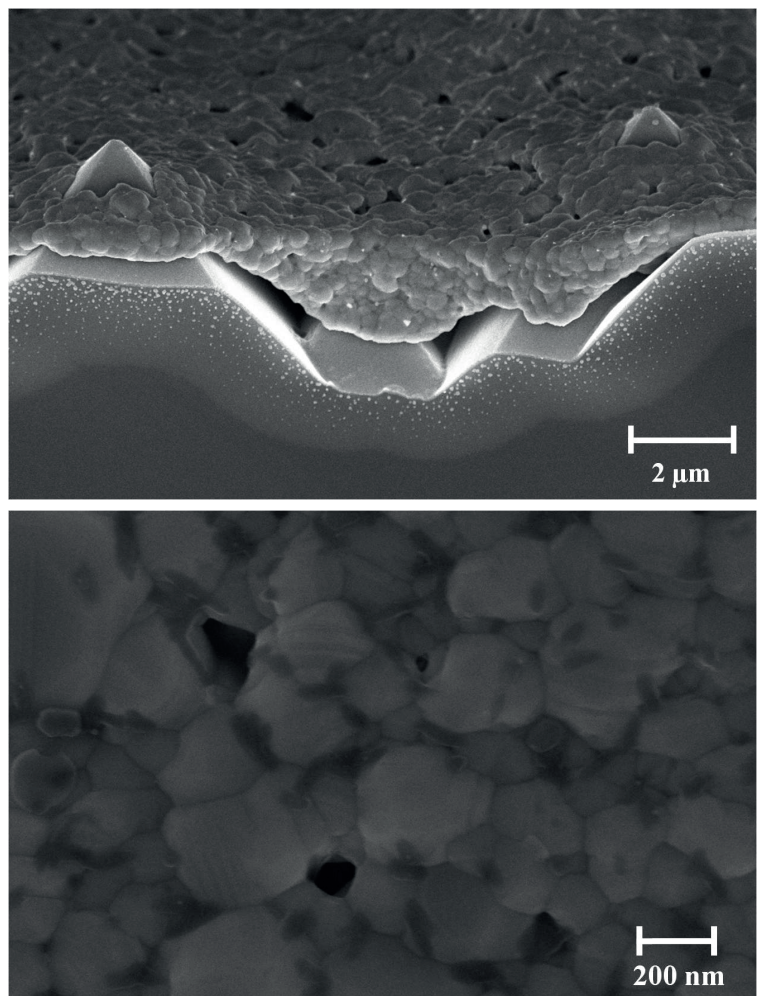

Fig. 16. SEM - images of the printed annealed conductive elements on the ITO surface which were annealed at temperature $120^{\circ} \mathrm{C}$ for 15 min: at the top - a side view, on the down - a top view. 


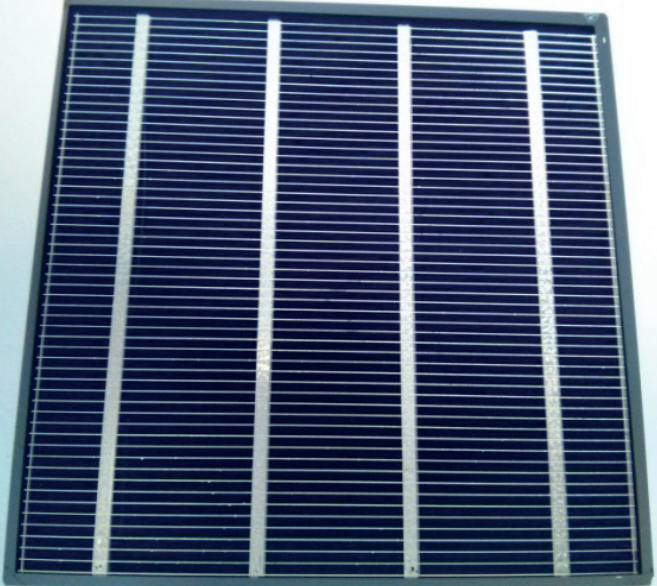

Fig. 17. Image of the printed conductive elements on the silicon substrate (by size $15 \mathrm{~cm} \times 20 \mathrm{~cm}$ ) covered by ITO layer.

exceed $2 \mu \mathrm{m}$ (Figure 17). The printed lines were annealed at temperature $120^{\circ} \mathrm{C}$ for $15 \mathrm{~min}$. The printed lines resistivity is on average $2 \mathrm{Om} / \mathrm{cm}$.

Thus, it was proved that the technology of ink-jet printing with the use of nano-ink on the basis of conductive nanoparticles may be used in perspective to create conductive circuit wiring on the substrates covered by the ITO layer, particularly on solar sells.

\subsection{Printing of the conductive lines} ON THE SILICON SURFACE WITH THE USE OF SILVER NANO-INK

As nowadays silicon is widely used in the electronic industry, the utterly important task

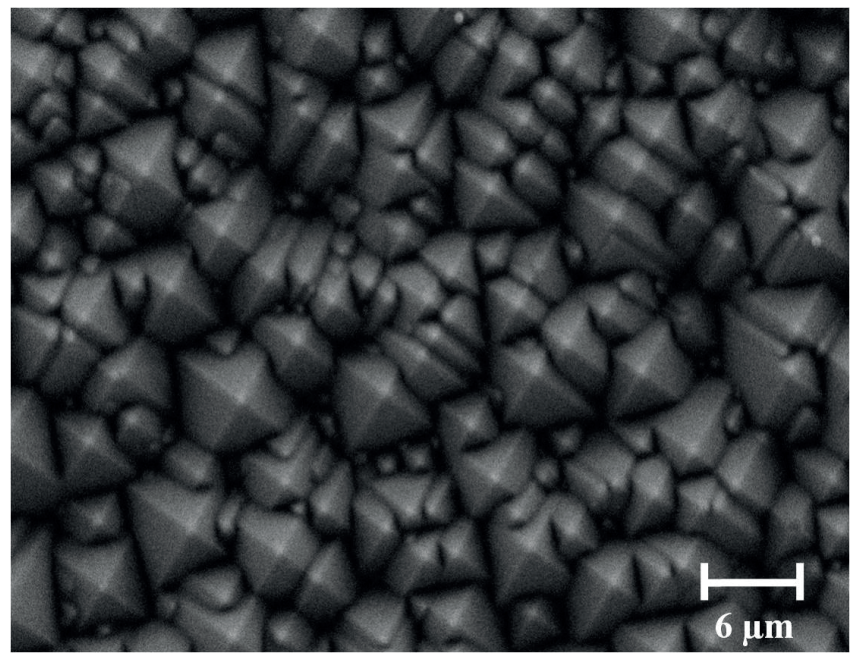

Fig. 18. SEM-image of the silicon substrate's surface

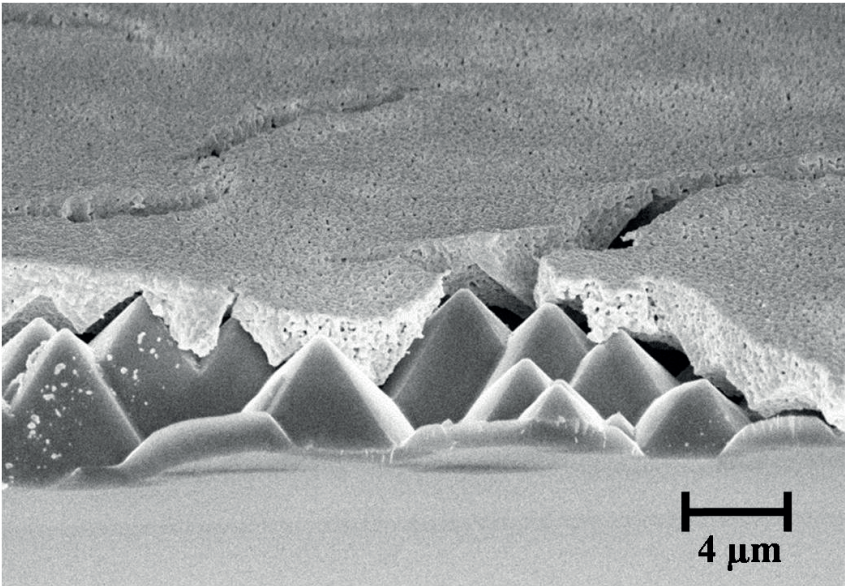

Fig. 19. SEM-image of the printed silver line on the silicon substrate (annealing temperature is $195^{\circ} \mathrm{C}, 60 \mathrm{~min}$ )

is testing of silicon substrates (Figure 18) in the technology of ink-jet printing of the electronic devices' components.

A number of experiments on silver nanoink printing were carried out with the use of Fujifilm Dimatix DMP - 2831 equipment on the silicon substrates' surface (Figures 19, 20).

The presented above images show that silver nano-ink sinters into the single structure after thermal treatment. This confirms the possibility to apply the present approach to produce electronic devices on the silicon substrates.
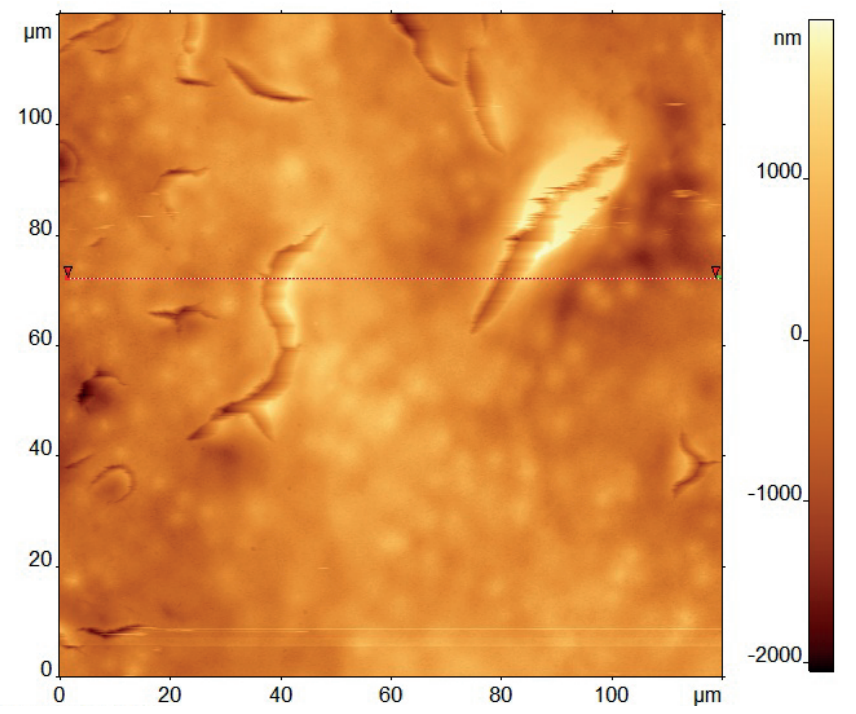

Fig. 20. AFM - image of the topology of the printed silver line's surface on the silicon substrate (annealing temperature is $195^{\circ} \mathrm{C}, 60$ min). 


\section{NANOSYSTEMS}

THE DISPERSIONS OF NANOPARTICLES IN WATER-ORGANIC SOLVENTS AS THE BASIS OF THE SILVER NANO-INKS...

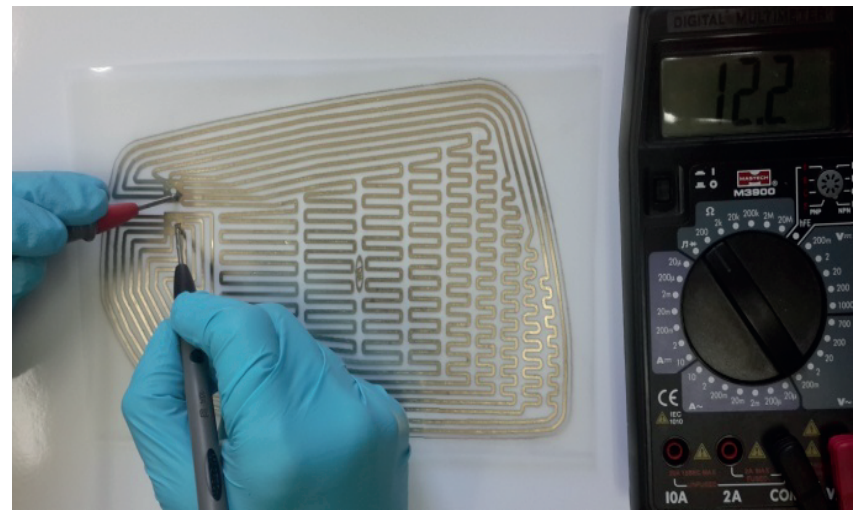

Fig. 21. Image of the sample - a heating element printed on the polymer film by silver nano-ink.

\subsection{Printing of the heating elements ON THE POLYMER FILM}

Ink-jet printing by metal-containing nanoink may be also applied to produce heating elements (Figure 21).

The experiments showed that such elements printed by synthesized silver nanoink had resistance $30 \mathrm{Om}$. It should be noted that maximum heating temperature was $50^{\circ} \mathrm{C}$ and $70^{\circ} \mathrm{C}$ at voltage $12 \mathrm{~V}, 18 \mathrm{~V}$ correspondingly.

\subsection{Printing of the Complex-geometry}

\section{CONDUCTIVE BOARDS ON DIFFERENT}

\section{SUBSTRATES}

Silver conductive boards were printed on the polymer films using the special high-precision ink-jet printer Fujifilm Dimatix 2831. It should be noted that the picture may have any geometry with the

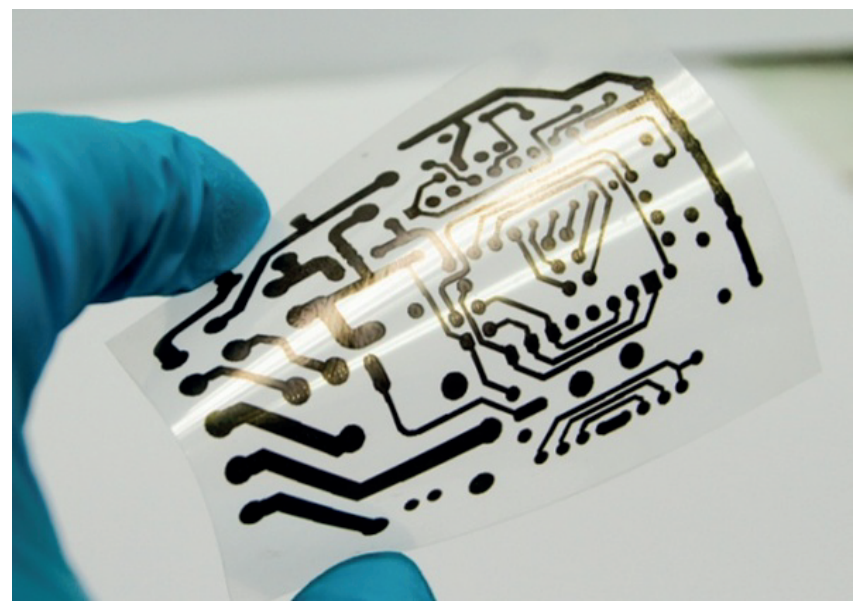

Fig. 22. Image of the conductive board printed using silver nano-ink on the polymer film.

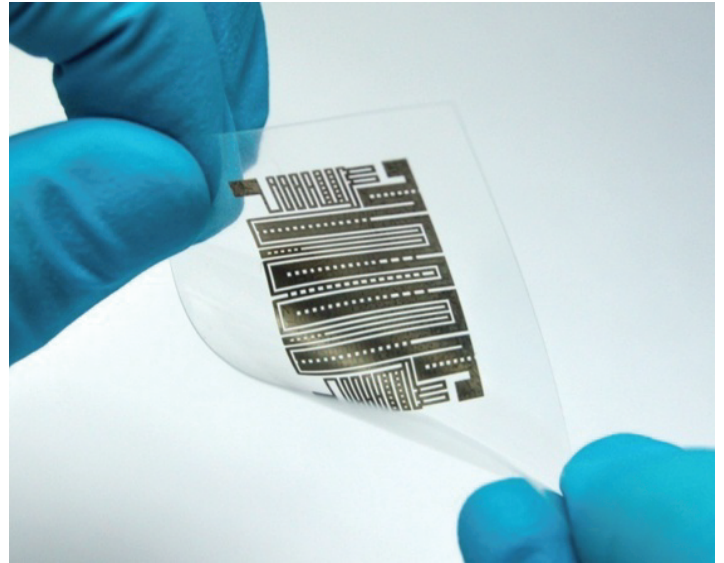

Fig. 23. Image of the conductive board printed using silver nano-ink on the polymer film.

resolution up to $20 \mu \mathrm{m}$ (Figures 22, 23). Conductivity, in turn, is defined by the number of the deposited substances and annealing temperature. Due to the melting of the flexible polymer films at temperature $200^{\circ} \mathrm{C}$, printing of conductive elements on polymer substrates is possible only using conductive nanoparticles, because their melting temperature is significantly lower than one of the massive analogs. Thus, the melting temperature of massive silver is $962^{\circ} \mathrm{C}$, but silver nanoparticles start sintering at the temperature $90^{\circ} \mathrm{C}$.

The model samples of the RFIDantenna (Radio Frequency IDentification) and conductive accumulator coverings were also printed (Figure 24) Polyimide film,

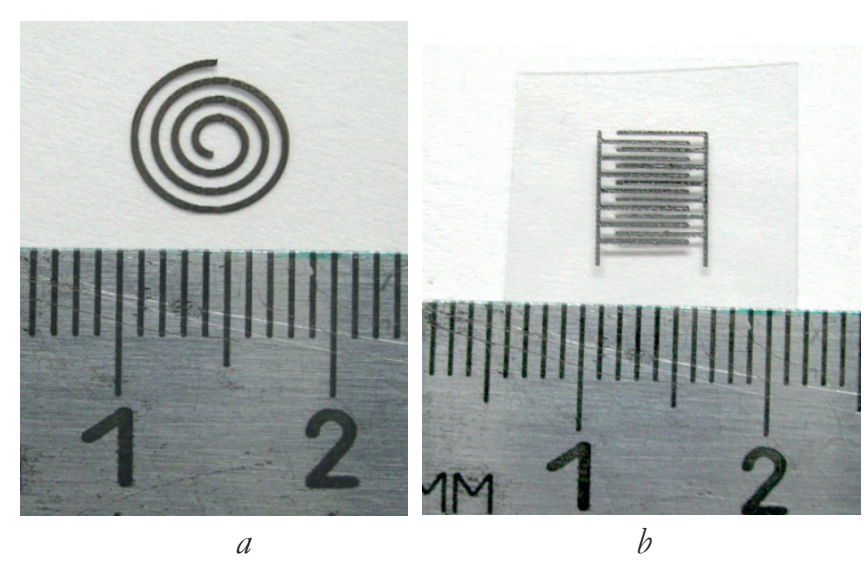

Fig. 24. Images of the RFID-antenna (a) and the conductive accumulator coverings (b) printed using silver nano-ink on the polymer film. 


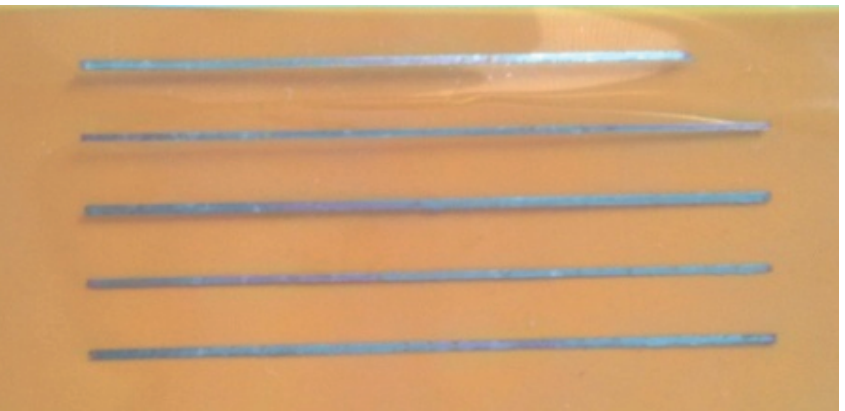

Fig. 25. Image of the conductive lines printed by silver nano-ink on the polyimide film.

ceramics, glazed paper, glass were also used as a substrate (Figures 25, 26).

The surface of glass and polyimide film was treated with solvents: isopropyl alcohol, acetone, chloroform and surface-active substances. Thus, the substrates' surface was degreased and got hydrophilic properties.

Nowadays ceramics is widely used in the electronic industry, mainly as a hard substrate of the electronic devices' elements. This is the reason for the choice of the ceramic surface. As ceramics has the porous structure, the adjacent conductive elements were short-circuited in ceramics structural pores. The problem was solved by supply of voltage which is needed to burn such "bridges". The possibility to solder to the printed structure was experimentally proved. This confirms the perspectives of realization into the existing technological cycles on production of the electronic devices' elements on ceramics.

Nowadays glazed paper is not used in electronic industry. However, the presented

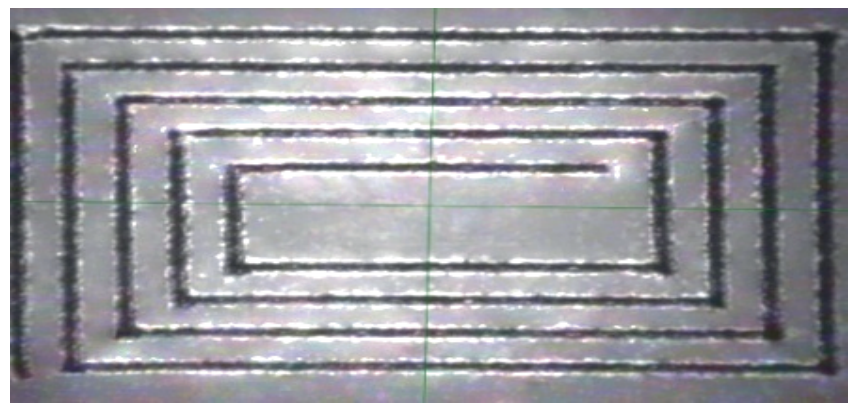

Fig. 26. Image obtained by the optical microscopy method of the RFID antenna by silver nano-ink on the polyimide film. substrate has one obvious advantage: due to its porous structure and the glazed layer on the surface, silver nano-ink' solvent is immediately absorbed into the structure, but silver nanoparticles remain on the surface. Thus, there is a possibility to draw, print conductive elements directly on paper without additional post-printing treatment.

\subsection{Printing on textile goods with the USE OF SILVER NANO-INK}

Printing on textile goods with the use of silver nano-ink was carried out (Figure 27). The presence of the final samples' electronic conduction is caused by continuous multiple coating consisted from the layers of the annealed metal nanoparticles on the cloth's surface. The stability of the sample's parameters to bending and interaction with water is unsatisfactory. The reason for this is crippling of the layer from annealed silver nanoparticles. The low adhesion degree is presumably caused by the significant number of nanoparticles' layers.

\section{CONCLUSION}

The "AkKo Lab" LLC company has developed the methodology of silver nano-ink production. In terms of colloid chemistry, the developed nano-ink

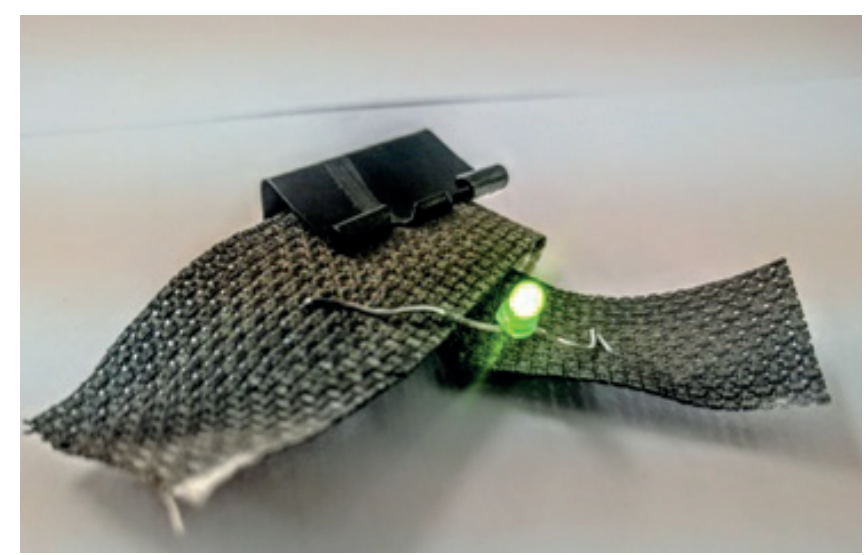

Fig. 27. Printing on textile goods with the use of silver nano-ink. 
represents highly concentrated dispersions of silver nanoparticles. According to the data of transparent electron microscopy and scanning electron microscopy, silver nanoparticles in nano-ink have the shape close to spherical one. Besides, silver nanoparticles are characterized by quite the narrow range of particles' distribution at sizes - from 8 to $12 \mathrm{~nm}$.

Silver nano-ink was adapted to print on the high-technology printer Dimatix Material Printer DMP-2831. Printing of different conductive structures on different nature substrates was worked through. They are ceramics, glass, ITO layer, flexible polymer films, textile goods. It was shown that printing quality depends on the wide range of the parameters: the substrate's nature, nano-ink adhesion to the substrate's surface, printing temperature, the number of printing passes.

It should be noted that printing is possible almost on any surface. However, to print successfully, it is necessary to treat printing surface beforehand by a physical (particularly by treatment in plasma) or a chemical method (treatment by surface-active substances, alcohols, etc.) in order to make the surface wet for nano-ink and suitable to print.

The important property of the developed nano-ink is quite low sintering temperature of silver nanoparticles. Thus, the sintering temperature of silver nanoink into the conductive layer is significantly lower than the melting temperature of its compact analog and accounts for about $100^{\circ} \mathrm{C}$.

Silver nano-ink developed by the "AkKo Lab" LLC company passed tests in the Fujifilm Dimatix Inc. company in California (the USA) and was highly estimated. Silver nano-ink is a commercial product and is supplied to the final customers in Russia and abroad.

The obtained results show the perspective to apply the technology of the ink-jet printing by the metal-containing nano-ink to produce the electronic devices, including those on flexible substrates.

This work was partly supported by the fund of assistance to innovation, project number 0016302.

\section{REFERENCES}

1. Kamyshny A, Steinke J, Magdassi S. Metalbased Inkjet Inks for Printed Electronics. The Open Applied Physics Journal, 2011, 4:19-36.

2. Finn DJ, Lotya M, Coleman JN. Inkjet Printing of Silver Nanowire Networks. ACS Appl. Mater. Interfaces, 2015, 7(17):9254-9261.

3. Torrisi F, Hasan T, Wu W, Sun Z, Lombardo A, Kulmala TS, Hsieh G-W, Jung SJ, Bonaccorso F, Paul PJ, Chu DP, Ferrari AC. Ink-Jet Printed Graphene Electronics. ACS Nano, 2012, 6(4):2992-3006.

4. Tobjork D, Osterbacka R. Paper electronics. Advanced materials, 2011, 23:1935-1961.

5. Gaikwad AM, Whiting GL, Steingart DA, Arias AC. Highly Flexible, Printed Alkaline Batteries Based on MeshEmbedded Electrodes. Advanced materials, 2011, 23:3251-3255.

6. Hildera M, Winther-Jensenb B, Clarka NB. Paper-based, printed zinc-air battery. Journal of Power Sources, 2009, 194:1135-1141.

7. Park J, Moon J, Shin H, Wang D, Park M. Direct-write fabrication of colloidal photonic crystal microarrays by ink-jet printing. Journal of Colloid and Interface Science, 2006, 298:713-719. 
184 SERGEY V. TKACHEV, VITALII P. KIM, ALEXEY E. KUSHNIR, DENIS. YU. KORNILOV, SERGEY P. GUBIN

8. Jillek W, Yung WKC. Embedded components in printed circuit boards: a processing technology review. International Journal of Advanced Manufacturing Technology, 2005, 25:350-360. 